\title{
Spin-filter state of Au-Co nanowires
}

\author{
Ekaterina Smelova, ${ }^{1,}$, Kseniya Tsysar ${ }^{1}$ and Alexander Saletsky ${ }^{1}$ \\ ${ }^{1}$ Lomonosov Moscow State University, Faculty of Physics, (Lomonosov MSU), 119991, Moscow, Russia
}

\begin{abstract}
Our theoretical study reveals the dependence of quantum conductance of Au-Co nanowires on their atomic structure. The results show the emergence of spin-filter state in one-dimensional Au-Co bimetallic nanowires. We found the existence of two transmission regime in Au-Co nanowires with low and high conductivity $1 \mathrm{G}_{0}$ and $2 \mathrm{G}_{0}$ for "zig-zag" and linear nanowire correspondingly. The study of transmission spectra of Au-Co nanowires reveals the control capability of spin transport regime by changing of bias voltage between bulk electrodes.
\end{abstract}

\section{Introduction}

Bimetallic atomic wires are the most promising objects for construction of new nanoelectronics and spintronics devices $[1,2]$. Bimetallic atomic nanowires possess the unique magnetic $[3,4,8,11]$ and conductive $[2,5]$ properties, i.e. spin polarized tunnel current, spinfiltering properties [5], giant magnetoresistance [2, 12] and giant magnetic anisotropy [11]. These properties of one-dimensional structures used now in leading laboratories of the world for the development of new storage or recording nanodevices. Recently IBM's labs (Yorktown, New York) by using CMOS technology produced the first prototype of racetrack memory based on "giant magnetoresistance" in vertical magnetic nanowires. By passing "spin polarized" currents through the data, the magnetic data can be moved up and down the skyscrapers which are vertical racetracks [6]. Thus the main problem of spintronics is the search for new spin control capabilities in one dimensional nanostructures [7].

\section{Method and model}

(a)

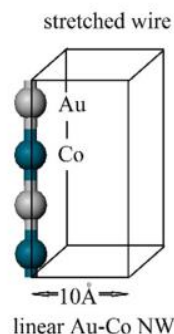

(b)

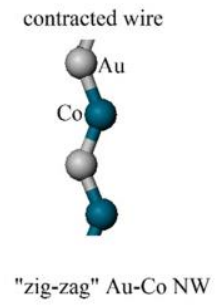

(c)

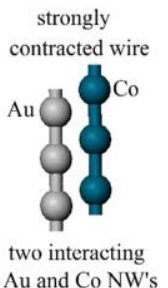

Fig.1 Model of Au-Co nanowire.

In the present paper the model of one dimensional atomic Au-Co nanowire is built in a full agreement with the result of experimental works on NC's formation in MCBJ technic [2,31] (Figure 1(a)). Under nanojunction formation with help of MCBJ technic at the last stage only one atomic chain formed between two massive electrodes with ballistic regime of quantum electron conductance through suspended atomic chain $[1,2]$. Our recent studies revealed the most stable configurations of bimetallic nanowires as evenly mixed bimetallic atomic chain $[9,10]$. We found that dimer formation in nanojunction $(\mathrm{NJ})$ chain leads to the destabilization of atomic bonds and as result to early breaking of NJ under stretching process $[9,13]$. Thus at the last stage of MCBJ or STM formation process only evenly mixed atomic nanowires form long conductive atomic bridges between two alloy $\mathrm{Au} / \mathrm{Co}$ electrodes, which emphasizes the quantum character of conductivity of nanocontacts, studied in the present work. Recent studies are focused on the dependence of transport properties of nanocontacts on the electrodes structure, atomic composition or magnetic ordering of bulk electrodes [1, $2]$. The atomic structure and magnetic properties of suspended atomic chain consciously have not been investigated however they can have the decisive impact on the electronic transport in the ballistic regime of bimetallic NJ. Moreover the electronic structure and magnetic properties of $\mathrm{NJ}$ chain strongly affect the spin polarised electron transport through a NJ. We offer an explanation of transport properties of bimetallic onedimentional structures with help of detail investigation of "inter-chain" interactions.

The study of the magnetic properties of the mixed Au-Co nanowires was carried out using the program Vienna Ab-initio Simulation Package (VASP) which is based on the density functional theory (DFT) [14]. The calculations presented in this paper were based on the self-consistent Kohn-Sham equations [15]. Electronic states were described using the basis of plane waves [16]. The cutoff energy for plane wave basis of $250 \mathrm{eV}$ was used. All calculations presented in this paper were performed using the generalized gradient approximation (GGA) and also local density approximation (LDA) for exchange-correlation

Corresponding author: smelova_e m@mail.ru 
functional and PAW (Projector Augmented-Wave) pseudopotentials (Perdew-Wang '91 treatment) [17, 18]. A structural relaxation was performed via a quasiNewton algorithm, using the exact Hellmann-Feynman forces acting on each atom [19]. The total energies of the system were converged up to $1 \mathrm{meV} / \mathrm{atom}$, while the residual force acting on each atom was less than 0.01 eV/A. The integration over Brillouin Zone (BZ) was performed using the tetrahedron method with Blöchl corrections. To calculate the total energy of the system we carried out the integration in reciprocal space by a special k-points grid of $1 \times 1 \times 7$ constructed according to k-point mesh in Monkhorst-Pack scheme [20]. The structure of mixed Au-Co nanowires (NWs) for magnetic calculations has been represented by means of three dimension supercell with periodic boundary conditions. Au-Co wire was represented by an atomic chain aligned along the $\mathrm{z}$ axis separated from its images along the $\mathrm{x}$ and $\mathrm{y}$ directions $(\sim 10 \AA)$ to avoid spurious interactions with them. A one-dimensional Brillouin zone was used, along the $\mathrm{z}$ axis of the wire. Mixed $\mathrm{Au}-$ Co nanowire was modelled as a four-atomic chain of two $\mathrm{Au}$ and two Co atoms, located along the $\mathrm{z}$ axis. To compare the calculated results for different bimetallic NWs (areas of existence, transmission, energies, etc.) for stretched or contracted mixed NWs, we consider them as the functions of an average interatomic distance. Average interatomic distance (d) for bimetallic NW represents the sum of all bond lengths in the wire projected on the wire axis ( $\mathrm{z}$ axis) divided by the number of atoms in the wire.

Transport calculations were carried out in the elastic limit, ballistic transport conductivity calculations were conducted within the Landauer-Buttiker formalism, where the (spin-dependent) conductance is given by (1) [21, 22]:

$G_{\sigma}=\frac{e^{2}}{h} \sum_{\sigma} \sum_{i j} T_{i j}^{\sigma}=\frac{e^{2}}{h} \sum_{\sigma} T^{\sigma}(E)=\frac{e^{2}}{h} \sum_{\sigma} \operatorname{Tr}\left[t_{\sigma}(E) t_{\sigma}^{+}(E)\right]=$ $\frac{G_{0}}{2} \sum_{\sigma} T^{\sigma}(E)$

Where $\sum_{\mathrm{ij}}$ indicates that the sum is performed over all channels Fermi energy (the open channels) and we have introduced the spin index $\sigma(\sigma=\uparrow, \downarrow$, spin direction), $T^{\sigma}(E)$-transmission coefficients $\left(T(E)=\sum_{\sigma} T^{\sigma}(E)\right)$, $t_{\sigma}(E)$-transmission coefficients of scattering matrix $\mathrm{S}$, which relates the wavefunction of the incoming and outgoing electrons with respect to the step potential, where $S=\left(\begin{array}{ll}r & t^{\prime} \\ t & r^{\prime}\end{array}\right)$ and $\mathrm{t}$ and $\mathrm{r}$ the transmission and reflection coefficients correspondingly for incoming waves from the left, whereas $t^{\prime}$, and $r^{\prime}$ are the counter parts for incoming waves from the right. We can clearly see that the conductance is written in terms of the conductance quantum $\mathrm{G}_{0}\left(1 \mathrm{G}_{0}=\frac{2 \mathrm{e}^{2}}{\mathrm{~h}}=\frac{1}{12.9} \mathrm{k} \Omega\right)$. The NEGF (non-equilibrium Green function) formalism for transport calculation [23-27] combined with densityfunctional theory and implemented in SMEAGOL, [1719] is used here to calculate the transmission coefficients (2):

$$
G=\frac{2 e^{2}}{h} \sum_{T r}\left[\Gamma_{L} G_{M}^{R+} \Gamma_{R} G_{M}^{R}\right]
$$

Where $G$-is total conductance, where transmission coefficients represented by (3):

$T(E)=\operatorname{Tr}\left[\Gamma_{L} G_{M}^{R+} \Gamma_{R} G_{M}^{R}\right](E)$

Where $G_{M}^{R}(E)$-retarded Green's function [22]. All quantities with the index $\mathrm{L}$ and $\mathrm{R}$ refer to the left and right electrodes respectively.

The CA (Ceperley/Alder) approximation (LDA) for the exchange-correlation potential is employed [28]. Troullier-Martins norm-conserving nonrelativistic and nonlocal pseudopotentials (PPs) are used for Au and Co. The atomic configurations for generating the PPs are $4 s^{2} 3 d^{7}(\mathrm{Co})$ and $5 d^{10} 6 s^{1}(\mathrm{Au})$. The cutoff radii (in atomic units) for $\mathrm{s}, \mathrm{p}$, and $\mathrm{d}$ components of the pseudopotential generation are respectively 2.6 a.u., 2.73 a.u. and 2.6 a.u. for $\mathrm{Au}$ and 2.35 a.u., 2.47 a.u. and 2.235 a.u. for Co. The $\mathrm{ab}$ initio engine behind SMEAGOL is the numerical implementation of DFT contained in the SIESTA (Spanish Initiative for Electronic Simulations with Thousands of Atoms) code [29, 30] which uses finite-ranged numerical orbitals as basis set. Splitvalence bases for Co are of double zeta type for $s, \mathrm{p}$ and $\mathrm{d}$ orbitals with the largest cutoff radii being 6.0 a.u. The electronic temperature is $300 \mathrm{~K}$. A mesh cutoff of 250 Ry, which specifies the real-space grid for defining the potential and the electron density, is used. Because $\mathrm{Au}$ atom has a large spin-orbit interaction we actually use calculation method which includes the effect of spinorbit coupling. This amendment to Hamiltonian is taken into account in the used software codes SIESTA and VASP.

\section{RESULTS}

We present in this paper results of our theoretical calculations of conducting properties of $\mathrm{Au}-$ Co nanowire and also the bias effect on the wire conductivity. Our atomic structure calculations predicted that mixed Au-Co nanowires are stable in large area of average interatomic distances $(1.6 \AA-3.0 \AA)$ and may possess a high structural stability at room temperature. Our ab-initio calculations have shown the transition of the Au-Co nanowire from linear configuration to a "zigzag" one under wire contraction up to $2.4 \AA$ (Fig.1 (b)). Further contraction of bimetallic nanowire leads to formation of two single component $\mathrm{Au}$ and $\mathrm{Co}$ nanowires strongly interacting with each other (Fig.1(c)). We calculated the conductance of $\mathrm{Au}-\mathrm{Co}$ nanowires in the whole range of average interatomic distances, corresponding to the area of the wire existence $(1.6 \AA$ $3.0 \AA$ ) including the region of two interacting $\mathrm{Au}$ and $\mathrm{Co}$ nanowires. These calculations simulate the real processes taking place in the chain of mixed Au-Co nanocontact during its formation in experiment by using MKBJ [2, 31] or STM [3, 4, 32] methods (Fig.1). We found the strong dependence of the total conductance of the wire on the "stretching-contraction" deformations emerging during the wire formation (see Fig.2, solid 
line). The total transmission of linear stretched nanowires (with average interatomic distance $>2.4 \AA$ ) was calculated about $2.0 \mathrm{G}_{0}$ and remains practically

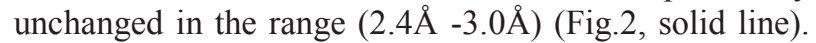
Under contraction wire goes into "zig-zag" configuration at average interatomic distance $\sim 2.4 \AA$. This structural transition of the wire leads to an abrupt decreasing of transmission value to $1 \mathrm{G}_{0}$ (Fig.2, solid line). When the system passes to two interacting wires (1.6 $\AA-2.0 \AA)$, the conductivity in each of the wires is $1 \mathrm{G}_{0}$, the total conductivity is $2 \mathrm{G}_{0}$ (distance between the two wires $\sim 2 \AA)$.

By the fact that Au-Co nanowires are magnetic and their magnetic properties strongly correlate with wire geometry we study in present paper the spin dependent electron transport in Au-Co nanowire [33, 34]. We found spin polarization of electron transport in contracted "zigzag" nanowires. However our study revealed the vanishing of spin polarization of electron transport in linear stretched Au-Co nanowire. Similar changes of conductivity were observed in our recent work in the Pt$\mathrm{Fe}$ nanowires [35] and in work of Nemnes et al. in graphene nanoribbons with magnetic impurities [36,37]. For quantitative estimation of the spin polarization of the electron transport through nanowire, we use a term degree of polarization [35] (4):

$$
g=\frac{G_{\uparrow}-G_{\downarrow}}{G_{\uparrow}+G_{\downarrow}}
$$

The value of $g$ drastically decreases under wire stretching to $\sim 0$ for linear nanowire (Fig.2, solid green line).

To study in detail the conductivity of bimetallic Au-Co nanowires we calculated the transmission spectra of linear and contracted "zig-zag" nanowires. Figure 3a illustrates the transmission spectra of the linear wire at average interatomic distance $2.5 \AA$. Our transmission spectra calculations have shown the absence of spin polarizations of electron transport in linear $\mathrm{Au}-\mathrm{Co}$ nanowires. We can see that transmission through spin-up and spin-down channels at Fermi level (Fermi energy of

(a)

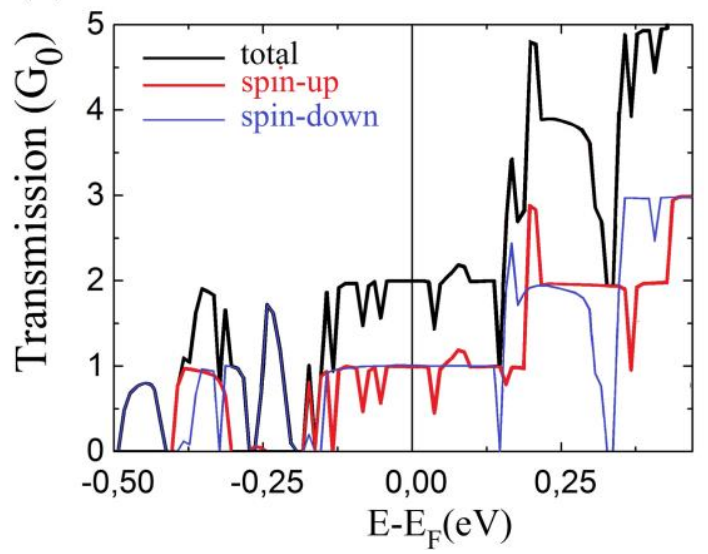

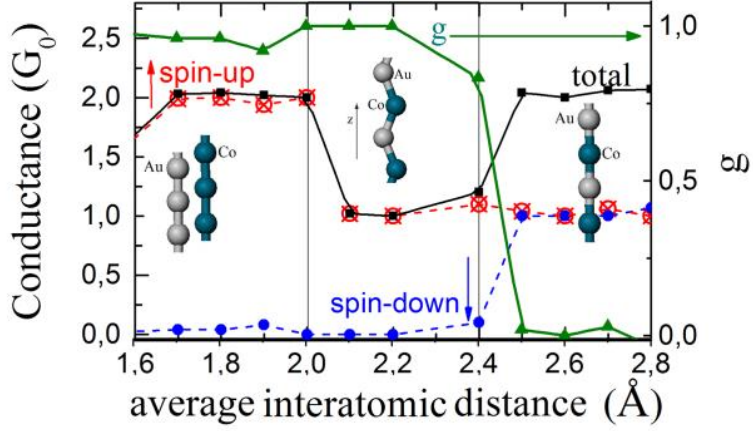

Fig. 2 Total quantum conductance of Au-Co nanowire (solid black line), spin-polarized conductance of spin-up (dashed red line) and spin-down (dashed blue line) channels and also the degree of spin polarization $(\mathrm{g})$ of quantum electron transport (solid green line) as a function of average interatomic distance. Insight figure represents the model of evenly mixed "zig-zag" Au-Co nanowire.

$\sim 1 \mathrm{G}_{0}$. Transmission pattern differs greatly for the contracted Au-Co nanowire. Figure 3b illustrates the partial transmission through spin-up and spin-down channels for average interatomic distance equals $2.2 \AA$. We can see that only transmission through spin-up channel is presented at Fermi level $\left(\sim 1 \mathrm{G}_{0}\right)$, the transmission through spin-down channel equals zero. The degree of spin polarization $(\mathrm{g})$ increases to 0.99 in "zig-zag" nanowire (see Fig.2, solid green line). Thus our transmission spectra calculations revealed that contracted "zig-zag" Au-Co nanowires represent the spin-filter system. We found also the dependence of spin conductance on the bias between bulk electrodes. Our transmission spectra calculations have shown that spin filtering state of Au-Co nanowire is metastable with narrow plateau of zero transmission spectra for one spin state at the Fermi level thus bias voltage about $0.06 \mathrm{eV}$ can destroy the spin filter state (Fig.3(b)) and put the system in non-polarized state (Fig.3(a)). As a result of our calculations we found the ability to control the transition point to the spin-filter state by changing of the bias between bulk electrodes. Our electronic structure calculations revealed the complex hybridized $\mathrm{s}-\mathrm{d}_{\mathrm{z}} 2-\mathrm{d}_{\mathrm{xz}}-$ $\mathrm{d}_{\mathrm{yz}}$ band formation between atoms in "zig-zag Au-Co

(b)

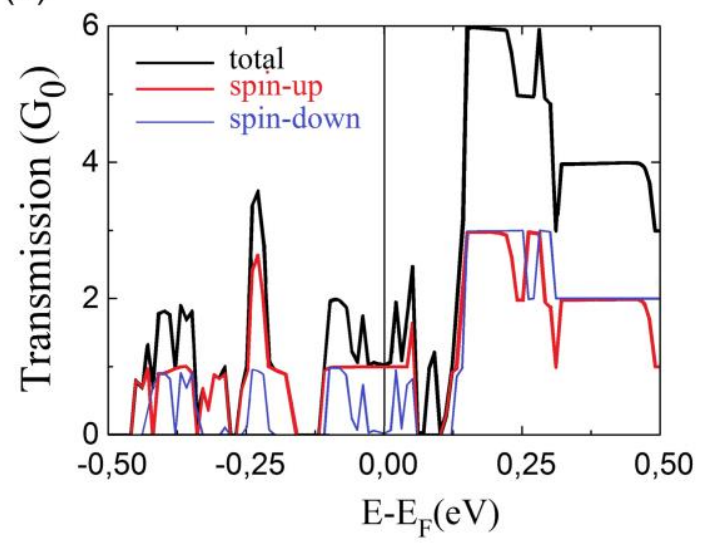

Fig. 3 Transmission $T(E)$ through spin-up and spin-down channel and total transmission at zero-bias of (a) linear Au-Co nanowire at average interatomic distance $2.5 \AA$ and (b) zig-zag nanowires at $2.2 \AA$ in spin filtering regime. $E_{F}$ is the Fermi energy of left and right hand side electrodes. 
coherent one-electron resonant tunnelling regime into a wire with two type of spin directions of electrons - spin up and spin down ones. The ratio of a length of a tunnelling area and the mean free path of an electron indicates that quantum interference of electrons exists in these systems (Au-37.7nm, Co-11.8nm) [38]. In case of linear nanowire each electron sequentially interact with magnetic and gold atoms and loss its original spin polarization as a result the quantum transport through the wire become non spin-polarised. In "zig-zag"

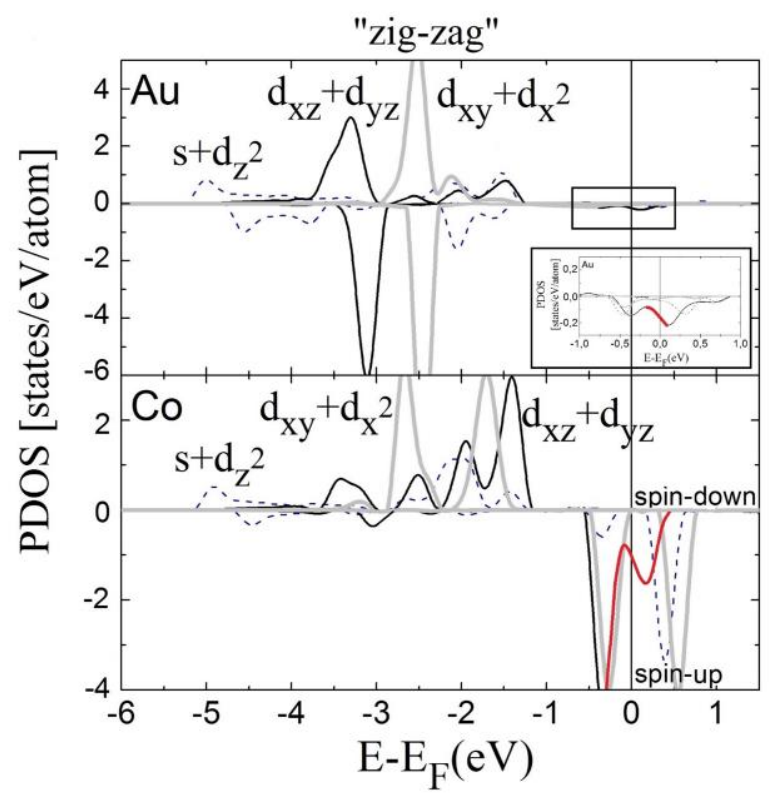

Fig. 4 Partial density of states for Au and Co atoms in "zigzag" Au-Co nanowire in spin-filter state at average interatomic distance $2.2 \AA$. Red line- $\mathrm{d}_{\mathrm{xz}}-\mathrm{d}_{\mathrm{yz}}$ band at the Fermi level for $\mathrm{Au}$ and Co atoms

configuration electrons propagate through the wire without the loss of spin polarisation thus electron may interact simultaneously with magnetic Co atoms (with spin up polarisation) and also with $\mathrm{Au}$ atoms in tunnel area. The strong exchange leads to appearance of nonzero phase difference for electrons with spin up and spin down polarisation as the result of interaction with magnetized cobalt atoms. The interference of these electron waves leads to the formation of a spin -filter state in one-dimensional "zig-zag" nanowires with preferred spin up direction of electron polarization.

\section{Conclusions}

The study revealed the dependence of quantum conductance of Au-Co nanowire on the elongation of the wire. We found the new spin-filter state in onedimensional Au-Co bimetallic nanowires. Moreover we found the existence of two transmission regimes in $\mathrm{Au}-$ Co nanowires with low and high conductivity $1 \mathrm{G}_{0}$ and $2 \mathrm{G}_{0}$ in "zig-zag" and linear nanowire correspondingly. We found the ability to control the conductivity of the wire by changing the geometry and length of the wire, as well as by applying an external voltage.

\section{Acknowledgements}

This work was supported by RFBR. The reported study was supported by the Supercomputing Center of Lomonosov Moscow State University and cluster MVS50K of the Interagency Supercomputer Center of the Russian Academy of Sciences.

\section{References}

1. A. Sokolov, Ch. Zhang, E. Y. Tsymbal, J. Redepending, B. Doudin, Nature Nanotechnology Lett. 2007, 2, 171 - 175 Quantum Wires Shik A.Ya. 1997 SOROSOVSKY EDUCATIONAL JOURNAL, 1997, No. 5,87-92.

2. H.-D. Chopra and S. Z. Hua, Phys. Rev. B 2002, 66, 020403. M. J. Lagos, F. Sato, P. A. S. Autreto, D. S. Galvao, V. Rodrigues, D. Ugarte, Nanotechnology 2010, 21, 485702.

3. J.Velev, R. F. Sabirianov, S. S. Jaswal, E.Y. Tsymbal, Phys. Rev. Lett. 2005, 94, 127203; J.C.Tung and G.Y.Guo, Phys. Rev. B 2010, 81, 094422.

4. V.S. Stepanyuk, P. Bruno, A.L. Klavsyuk et al. Phys. Rev. B 2004, 69, 3, 033302 ; T. Haug, K. Perzlmaier, and C. H. Back, Phys. Rev. B 2009, 79, 024414; K. M. Smelova, D. I. Bazhanov V.S. Stepanyuk et al. Phys. Rev. B 2008, 77, 3, 033408.

5. K. M. Tsysar, D. I. Bazhanov and A. M. Saletsky, Phys. Rev. B 2011, 84, 08545.

6. S.P. Parkin, M. Hayashi, L. Thomas, Science 2008, 320, 5873, 190-194.

7. N. Müller, W. Eckstein, W. Heiland, and W. Zinn, Phys. Rev. Lett. 1972, 29, 1651.

8. J.Bettini, F.Sato, P.Z.Coura, S.O.Danatas, D.S.Galvao, D.Ugarte, Nature Nanotechnology 2006, 1, 182 .

9. E. M. Smelova, K. M. Tsysar', D. I. Bazhanov and A. M. Saletsky, JETP Lett. 2011, 93, 3, 129-132; K. M. Tsysar', E. M. Smelova, D. I. Bazhanov and A. M. Saletsky, JETP Lett. 2011, 94, 3, 228-232.

10. K.M.Tsysar, D.I. Bazhanov, A.M. Saletsky, V.S. Stepanyuk, and W.Hergert, Phys. Solid State 2010, 52, 3, 641. 11. E.M. Smelova, A.L. Klavsyuk, K.M. Tsysar, A.M. Saletsky, Moscow University Physics Bulletin, 2013, 68, 1, 92-95.

12. S. Egle, C. Bacca, H. - Fr. of Pernau, M. Hafner, D. Hinzke, U. Nowak, E. Scheer, Phys. Rev. B 2010, 81, 134402

13. K.M.Tsysar, D.I.Bazhanov, E.M.Smelova, and A.M.Saletsky Appl. Phys. Lett. 2012, 101, 043108.

14. G. Kresse and J. Furthmuller, Phys. Rev. B 1996, 54 , 11169.

15. W. Kohn, L.J. Sham, Phys. Rev. A 1965, 140, 1133-1138. 16. J. Hafner, G. Kresse, A. Eichler, R. Lorentz, R. Hirschl, M. Marsman, Vasp workshop, http://cms.mpi.univie.ac.at/vaspworkshop/slides/documentation.htm.

17. J.P. Perdew, J.A. Chevary, S.H. Vosko, K.A. Jackson, M.R. Pederson, D.J.Singh, C. Fiolhais, Phys. Rev. B 1992, 46, 66716687; M. C. Payne, M. P.Teter, D. C. Allan, T.A. Arias, J.D. Joannopoulos, Rev. Mod. Phys. 1992, 64, 4, 1045-1097; M. C. Payne, et al., Rev. Mod. Phys. 1992, 64, 1045-1077.

18. P P. Blochl, Phys. Rev. B 1994, 50, 17953.

19. P. N. Molin, Solid State Storage of Hydrogen in Magnesium Alanate - a Density Functional Study, Department of Physics University of Oslo, (2005).

20. H. J. Monkhorst and J. D. Pack, Phys. Rev. B 1976, 13, 5188.

21. R. Landauer, Philos. Mag. 1970, 21, 863.

22. Theoretical and Computational Aspects of Electronic Transport at the Nanoscale, Alexandre Reily Rocha 2007, Trinity College. 
23. L. P. Kadanoff and G. Baym, Quantum Statistical Mechanics_Benjamin/Cummings, 1962, New York.

24. L. V. Keldysh, Sov. Phys. JETP 1965, 20, 1018.

25. Y. Meir and N. S. Wingreen, Phys. Rev. Lett. 1992, 68, 2512.

26. S. Datta, Electronic Transport in Mesoscopic Systems Cambridge University Press, 1995, Cambridge, England.

27. H. Haug and A. P. Jauho, Quantum Kinetics in Transport and Optics of Semiconductors Springer-Verlag, 1996, Berlin. 28. N. Troullier and J. L. Martins, Phys. Rev. B 1991, 43.

29. P. Ordejon, E. Artacho, and J. M. Soler, Phys. Rev. B 1996, 53, R10441.

30. J. Soler, E. Artacho, J. D. Gale, A. Garcia, J. Junquera, P. Ordejon, and D. Sanchez-Portal, J. Phys.: Condens. Matter 2002, 14, 2745.

31. M. Czerner, A. Bagrets, V. S. Stepanyuk, A. L. Klavsyuk, and I. Mertig Phys. Rev. B, 2006, 74, 115108.

32. A.L. Klavsyuk, A.M. Saletsky, Physics-Uspekhi 58, 933 (2015); A.L. Klavsyuk, S.V. Kolesnikov, E.M. Smelova, A.M. Saletsky, JETP Lett., 2010, 91, 158.

33.Tsysar K.M., Bazhanov D.I., Smelova E.M., Saletsky A.M., Applied Physics Letters, American Institute of Physics (United States), 2012,101, 043108-043112.

34. Smelova E.M., Tsysar K.M., Bazhanov D.I., Saletsky A.M., Letters to the "Journal of Experimental and Theoretical Physics", 2011, 93, № 3, 139-142.

35. E.M. Smelova, K.M. Tsysar, A.M. Saletsky, Physical Chemistry Chemical Physics, 2014, 16, 8360 .

36.G.A.Nemnes, S.Antohe, Materials Science and Engeniring B, 2013,178, 1347.

37. G.A.Nemnes, Journal of Nanomaterials, 2013, 408475

38. Daniel Gall, JOURNAL OF APPLIED PHYSICS, 2013, $119,085101$. 